\title{
Micotoxinas em alimentação animal no período de maio/1997 a março/2001 no Laboratório de Toxicologia Veterinária da Universidade Estadual de Londrina - Londrina - PR
}

\section{Micotoxins in animal feed detected from May/1997 to March/2001 at the Veterinary Toxicology Laboratory of State University of Londrina - Londrina - Paraná State}

\author{
Daisy Pontes Netto ${ }^{1 *}$; Aline Tramontini Zanluchi²; \\ Marcia Sassahara'; Erika Kubota Yanaka ${ }^{3}$
}

\section{Resumo}

As micotoxinas são metabólitos secundários tóxicos que não têm significado bioquímico no crescimento e desenvolvimento fúngico. Entre as recomendações para controle de micotoxinas está o monitoramento constante, por meio de um programa de colheita de amostras e análises das micotoxinas. Analisaram-se 436 amostras no período de maio/1997 a março de 2001 para detecção das seguintes micotoxinas: aflatoxinas B1, B2, G1 e G2, zearalenona e ocratoxina A. Utilizou-se a técnica de Cromatografia em Camada Delgada (CCD). Verificou-se que, a maioria das análises efetuada foi de grãos e silagem de milho, 273 amostras, representando 62,6\% do total (436), este número pode estar ligado ao tipo de produção animal, vacas holandesas, que mais utilizam este tipo de alimentação, já que a maioria das amostras foi proveniente de propriedades leiteiras da região norte do Paraná. Observouse que a micotoxina mais detectada foi a zearalenona com $57,5 \%$ de amostras positivas e representando $28 \%$ do total de substratos. As aflatoxinas representaram 36,3\%, no total de amostras positivas e 17,7\% dos substratos. Quando se correlacionou o número de amostras enviadas e a positividade, observamos que no período do verão e do outono houve maior ocorrência de análises positivas, 64,3 e 73,5\% respectivamente. Os resultados obtidos refletem o tipo de produção animal que temos em nossa região e as atividades dos fenômenos climáticos que ocorreram nos anos de 1997 a 2001, com chuvas intensas, variações abruptas de temperatura, facilitando o desenvolvimento de fungos e conseqüentemente o aparecimento de micotoxinas.

Palavras chave: Micotoxinas; Alimentos de Uso Animal; Aflatoxinas; Zearalenona

\footnotetext{
Abstract

Micotoxins are toxic secondary metabolites that have no biochemical significance in fugal growth and development. Recommendations for micotoxin control include constant monitoring by a program of harvest samples and micotoxin analysis. A total of 436 samples were analyzed from May 1997 to March 2001 to detect the following micotoxins: aflatoxins B1, B2, G1 and G2, zearalenona and ocratoxina A. The thin layer chromatography technique was used (CCD). Most of the analyses performed were of

1 Laboratório de Toxicologia Veterinária, Departamento de Medicina Veterinária Preventiva/CCA - Universidade Estadual de Londrina - UEL. E-mail: rnetto@uel.br.

2 Alunas do Programa de Pós Graduação em Sanidade Animal (nível: Mestrado), DMVP/CCA/UEL.

3 Aluna Estagiária do Laboratório de Toxicologia Veterinária, DMVP/CCA/UEL

* Autor para correspondência.
} 
maize grains and silage, 273 samples, representing $62.6 \%$ of the total (436) and this number may be linked to the type of animal production, Holstein cows, that most use this type of food, as most of the samples came from dairy farms in the north of Paraná state. Zearalenona was the most detected micotoxin with $57.5 \%$ positive samples representing $28 \%$ of all the substrates. The aflatoxin represented $36.3 \%$ of the total of positive samples and $17.7 \%$ in the substrates. When the number of samples received for analysis and positiveness are correlated, it was observed that there was a greater occurrence of positive analyses in the summer and autumn, $64.3 \%$ and $73.5 \%$, respectively. The results obtained reflect the type of animal production in this region and the climatic phenomena that occurred 1997-2001 such as heavy rainfall and abrupt temperature variations that eased fungus development and consequently the appearance of micotoxins.

Key words: Micotoxins; Animal feed; Aflatoxins; Zearalenona

As micotoxinas são produzidas principalmente por estruturas miceliais de fungos filamentosos, ou mais especificamente, pelos fungos. As micotoxinas são metabólitos secundários que não têm significado bioquímico no crescimento e desenvolvimento fúngico (MOSS, 1991). Algumas micotoxinas são de interesse de saúde pública e agronômica tais como: aflatoxinas, ocratoxinas, tricotecenos, zearalenona, fumonisinas, toxinas tremorgênicas e alcalóides de ergot. Estas micotoxinas computam milhões de dólares, anualmente, em perdas mundiais na saúde humana, animal e em produtos agrícolas (SHANE, 1994; VASANTHI; BHAT, 1998).

A indústria brasileira de alimentação animal obteve uma receita bruta de \$US 6,8 milhões em 1999 em vendas de 32,5 milhões de toneladas de alimentos: $59,2 \%$ em alimentos para aves, $29 \%$ para suínos, $6,4 \%$ para gado bovino e $5,4 \%$ para outros tipos de animais (NATIONAL TRADE UNION OF ANIMAL FEED INDUSTRIES, 2000).

Em 1993, a World Human Organization International Agency for Research on Cancer (WHOIARC) avaliaram o potencial carcinogênico da aflatoxina, ocratoxina, tricotecenos, zearalenona e fumonisinas. A aflatoxina com contaminação por produtos não experimentais foi classificada como carcinogênico para humanos (Grupo 1), enquanto a ocratoxina e a fumonisina foram classificadas como possíveis carcinogênicos (Grupo 2). Os tricotecenos e a zearalenona, não foram classificados como carcinogênicos para humanos (Grupo 3). As micotoxinas afetam diversos órgãos no organismo animal. A aflatoxina, por exemplo, afeta o fígado e os rins e pode causar mutações genéticas (MULRENNAM, 1999).

Os fungos consomem grande parte da energia e proteína armazenada nos grãos. Uma carga de milho severamente afetado pode perder até $10 \%$ de sua energia metabolizável e 5\% de sua proteína. Além disso, as micotoxinas deterioram os grãos ou a ração, reduzindo sua palatabilidade. A deterioração fúngica provoca danos no germe dos grãos, descoloração, alteração nutricional e perda de matéria seca. (LAZZARI, 1997).

A produção de milho no Brasil foi estimada em 32,7 milhões de toneladas para o ano 2000 dos quais, $65 \%$ foram destinadas para a alimentação animal (SALAY; MERCADANTE, 2002). As forragens representam 65 a $80 \%$ da dieta fornecida a ruminantes em grande parte do mundo. Assim, a qualidade da forragem afeta de maneira significativa o rendimento leiteiro, ganho de peso, eficiência alimentar e outros aspectos do desempenho de ruminantes (BOLSEN, 1999).

A contaminação por micotoxinas é difícil de se evitar, pois existem inúmeros fatores que atuam em conjunto, favorecendo a contaminação da cultura ou da forragem por fungos e à medida que ocorrem alterações do padrão climático mundial, fenômenos do tipo el niño e la niña, são encontrados fungos produtores de micotoxinas em áreas consideradas anteriormente livres destes patógenos (MABBETT, 1999). Os fatores que favorecem o desenvolvimento do fun- 
go são a umidade, temperatura, período de armazenamento, nível de contaminação, presença de impurezas e insetos, nível de oxigênio e as condições físicas e sanitárias da semente ou do grão (LOPEZ; CHRISTENSEN, 1967).

A necessidade de regulamentos que estabeleçam limites máximos de tolerância de micotoxinas em alimentos e rações é geralmente, reconhecida nos países desenvolvidos enquanto muitos países em desenvolvimento não possuem nenhuma legislação. Dentre as recomendações práticas para controle de micotoxinas (aflatoxinas, ocratoxinas, zearalenona, toxina T-2, vomitoxina, fumonisina) está o monitoramento constante de grãos ou da própria planta, por meio de análises laboratoriais. Este monitoramento é o ponto fundamental num programa de controle de micotoxinas (SABINO, 1999).

Sem informações toxicológicas não se pode determinar se a substância em questão induz perigo ou não e para isso, é necessário monitorar os níveis de contaminação por micotoxinas dos produtos, subprodutos e derivados de origem vegetal e colaborar com as ações de assistência técnica visando à prevenção e controle de micotoxinas nas fases de cultivo, colheita e pós-colheita (SABINO, 1999).

Em 1999, o Brasil possuía 151 milhões de cabeças de gado, 314 milhões de pintinhos e 37 milhões de suínos. As micotoxinas causam uma variedade de problemas de saúde nos animais reduzindo a eficiência econômica das indústrias (SALAY; MERCADANTE, 2002).

Existem diversas técnicas que podem auxiliar os fabricantes e os produtores a monitorarem a presença de fungos e micotoxinas. Os testes variam desde técnicas altamente sofisticadas que utilizam Cromatografia Líquida de Alto Desempenho (HPLC) a testes rápidos que podem ser feitos na granja ou no moinho de ração.

Este trabalho teve com objetivo compilar informações para auxiliar no monitoramento da presença de micotoxinas em alimentos fornecidos aos animais e a relação com a sazonalidade regional em seu de- senvolvimento. O levantamento das análises foi realizado no Laboratório de Toxicologia Veterinária do Departamento de Medicina Veterinária Preventiva da Universidade Estadual de Londrina (UEL), Londrina, Pr, no período de maio de 1997 a março de 2001. As amostras analisadas foram colhidas em propriedades do estado do Paraná, São Paulo, Santa Catarina, Rio Grande do Sul, Mato Grosso, Mato Grosso do Sul, Minas Gerais e Goiás e enviadas ao laboratório para análise. Foram analisadas 436 amostras no período de maio de 1997 a março de 2001 para detecção das seguintes micotoxinas: aflatoxinas B1, B2, G1 e G2, zearalenona e ocratoxina A.

Utilizou-se a técnica para determinação de aflatoxinas, ocratoxina e zearalenona por Cromatografia em Camada Delgada (CCD) de acordo com metodologia de Soares e Rodriguez-Amaya (1989) adaptada pelo Instituto Adolfo Lutz (1996). Os padrões (Sigma Inc. - EUA) utilizados para a técnica de $\mathrm{CCD}$, nas concentrações de 2,25, 2,62, 2,45, 4,55 e $68 \mathrm{mg} / \mathrm{mL}$, foram preparados conforme metodologia da Association of Official Analytical Chemists (AOAC) adaptada pelo Instituto Adolfo Lutz (1996) e os limites de detecção do método foram: 2,5 e $55 \mathrm{mg} / \mathrm{Kg}$ e o limite de determinação 4, 10 e $165 \mathrm{mg} / \mathrm{Kg}$ para aflatoxina B1, ocratoxina A e zearalenona, respectivamente. Para a cromatografia foram utilizadas placas de alumínio, cobertas com sílica gel 60G, tamanho 20x20 e 0,25mm de espessura ativadas antes do uso a $110^{\circ} \mathrm{C}$ por 60 minutos. A leitura das placas foi feita com aparelho com lâmpada ultravioleta de 15 watt e comprimento de onda de 235 e 360nm. Para confirmação das aflatoxinas utilizou-se a reação com ácido trifluoroacético (INSTITUTO ADOLFO LUTZ, 1996).

Os resultados obtidos no levantamento de dados do Laboratório de Toxicologia Veterinária foram tabulados pela positividade das amostras, distribuição e ocorrência de cada micotoxina nas estações do ano, distribuição nos anos estudados e positividade por tipo de substrato analisado.

Na Tabela 1 verifica-se que grande parte das análises efetuadas no período analisado constituiu-se, 
principalmente, de grãos e silagem de milho, foram analisadas 273 amostras representando $62,6 \%$ do total recebido (436) no laboratório, este número pode estar ligado ao tipo de produção animal, vacas ho- landesas, que mais utilizam este tipo de alimentação, já que a maioria das amostras foi proveniente de propriedades leiteiras da região norte do Paraná.

Tabela 1 - Positividade das amostras enviadas para análise em Cromatografia em Camada Delgada (CCD), no período de maio/1997 a março/2001, de acordo com o produto e tipo de micotoxina.

\begin{tabular}{lrrrrrrrrr}
\hline $\begin{array}{c}\text { Produto/ } \\
\text { micotoxina }\end{array}$ & $\begin{array}{c}\mathrm{n}^{\mathbf{0}} \text { de } \\
\text { amostras }\end{array}$ & Aflatoxinas & \multicolumn{1}{c}{ Zearalenona } & $\%$ & Ocratoxina & $\%$ & $\begin{array}{c}\text { Total } \\
\text { positivo }\end{array}$ & $\%$ \\
\hline soja & 21 & 3 & 14,3 & 9 & 42,9 & 1 & 4,8 & 13 & 61,9 \\
silagem & 133 & 25 & 18,8 & 52 & 39,1 & 4 & 3,0 & 81 & 60,9 \\
milho & 140 & 15 & 10,7 & 26 & 18,6 & 6 & 4,3 & 47 & 33,6 \\
rações & 74 & 23 & 31,1 & 13 & 17,6 & 1 & 1,3 & 37 & 50,0 \\
trigo & 17 & 0 & 0 & 3 & 17,6 & 0 & 0 & 3 & 17,6 \\
amendoim & 5 & 5 & 100 & 5 & 100 & 0 & 0 & 10 & 100 \\
girassol & 8 & 0 & 0 & 8 & 100 & 0 & 0 & 8 & 100 \\
algodão & 9 & 3 & 33,3 & 2 & 22,2 & 0 & 0 & 5 & 55,5 \\
variadas & 29 & 3 & 10,3 & 4 & 13,8 & 1 & 3,5 & 8 & 27,6 \\
\hline Total & 436 & 77 & 17,7 & 122 & 28,0 & 13 & 3,0 & 212 & 48,7 \\
\hline
\end{tabular}

Destas amostras, observa-se que a micotoxina mais detectada foi a zearalenona, com 122 análises indicando 57,5\% das amostras positivas e representando $28 \%$ do total de substratos. Talvez por ter ação mais evidente, alterando os ciclos reprodutivos dos animais e levando a perdas econômicas importantes para o produtor fazendo com que ele busque diagnosticar o problema em seu rebanho. Observou-se também que as aflatoxinas têm um número significativo, 77 análises representando 36,3\%, no total de amostras positivas e de $17,7 \%$ dos substratos.
As distribuições das amostras de acordo com as estações do ano são visualizadas na Tabela 2. Observa-se que o maior número de amostras enviadas foram nas estações do inverno e da primavera e verifica-se que foram analisadas em maior quantidade silagens e grãos de milho, refletindo a época em que se utiliza este tipo de alimentação para os bovinos leiteiros, que é a grande parte das propriedades que procuram monitoramento laboratorial para diagnóstico da presença de micotoxinas.

Tabela 2 - Tipos de amostras recebidas durante o período de maio de 1997 a março de 2001 e sua distribuição nas diferentes estações do ano.

\begin{tabular}{lrrrrrrrrrr}
\hline $\begin{array}{c}\text { Estações do } \\
\text { ano/amostras }\end{array}$ & soja & silagem & milho & rações & amendoim & trigo & girassol algodão & outras & Total \\
\hline verão & 1 & 13 & 4 & 12 & 5 & 1 & 8 & 4 & 8 & 56 \\
outono & 3 & 34 & 11 & 23 & 0 & 1 & 0 & 1 & 10 & 83 \\
inverno & 10 & 51 & 87 & 17 & 0 & 3 & 0 & 1 & 4 & 173 \\
primavera & 7 & 35 & 38 & 22 & 0 & 12 & 0 & 3 & 7 & 124 \\
\hline Total & 21 & 133 & 140 & 74 & 5 & 17 & 8 & 9 & 29 & 436 \\
\hline
\end{tabular}


Na Tabela 3 observa-se a distribuição das amostras positivas e a ocorrência das micotoxinas em diferentes estações do ano, constatando-se maior ocorrência da zearalenona independente da época. Quando se correlaciona a presença das micotoxinas com as estações do ano, verifica-se que houve uma maior ocorrência de análises positivas durante o período do inverno, em que foi enviado maior número de amostras e conseqüentemente maior positividade, mas se formos correlacionar o número de amostras enviadas e a positividade, independente do tipo de micotoxina, observamos que no período do verão e do outono houve maior ocorrência de análises positivas, 64,3 e $73,5 \%$ respectivamente, talvez por que estas épocas são de maior índice pluviométrico e com temperaturas mais elevadas aumentando a possibilidade do aparecimento de fungos e conseqüentemente de micotoxinas.

Tabela 3 - Número e porcentagens de análises positivas para aflatoxinas, zearalenona e ocratoxinas, distribuídas de acordo com ocorrência nas estações do ano durante o período de maio de 1997 a março de 2001.

\begin{tabular}{lccrrrrrr}
\hline $\begin{array}{c}\text { Estações do } \\
\text { ano/micotoxina }\end{array}$ & Aflatoxina & $\%$ & Zearalenona & $\%$ & Ocratoxina & $\%$ & Total & $\%$ \\
\hline Verão & 13 & 36,1 & 23 & 63,9 & 0 & 0 & 36 & 17,0 \\
Outono & 23 & 37,7 & 34 & 55,7 & 4 & 6,6 & 61 & 28,8 \\
Inverno & 25 & 35,7 & 41 & 58,6 & 4 & 5,7 & 70 & 33,0 \\
Primavera & 16 & 35,6 & 24 & 53,3 & 5 & 11,1 & 45 & 21,2 \\
\hline Total & 77 & 36,3 & 122 & 57,5 & 13 & 6,1 & $\mathbf{2 1 2}$ & \\
\hline
\end{tabular}

Na Tabela 4 observa-se a distribuição das amostras positivas de acordo com o ano e estação climática. Estes são condizentes com a literatura que cita como melhor desenvolvimento das micotoxinas em climas mais úmidos, em torno de $65 \%$ e temperaturas alternadas entre 22 a $27^{\circ} \mathrm{C}$ e 12 a $15^{\circ} \mathrm{C}$, que ocorreram durante o ano de 1998 nos períodos do outono e inverno, observa-se também o mesmo comportamento no inverno do ano de 1999 em que houve grandes variações de temperatura num mesmo período, conforme dados do Instituto Agronômico do Paraná (2001).

Tabela 4 - Distribuição das análises positivas por ano e por estação durante o período de maio de 1997 a março de 2001.

\begin{tabular}{llllll}
\hline & verão & outono & inverno & primavera & Total \\
\hline 1997 & - & - & 1 & 4 & 5 \\
1998 & - & 38 & 33 & 23 & 94 \\
1999 & 8 & 19 & 33 & 13 & 73 \\
2000 & 1 & 4 & 4 & 4 & 13 \\
2001 & 27 & 0 & 0 & 0 & 27 \\
\hline Total & 36 & 61 & 71 & 44 & $\mathbf{2 1 2}$
\end{tabular}

Os resultados globais destas análises refletem o tipo de cultura agronômica que temos em nossa região em que se utiliza com freqüência o plantio direto e somando-se ás atividades dos fenômenos climáticos que ocorreram nos anos de 1997 a 2001, com chuvas intensas, variações abruptas de temperatura, facilitando o desenvolvimento de fungos e conseqüentemente o aparecimento de micotoxinas.

No Brasil o Ministério da Agricultura e Abastecimento define valores de 20 ppb para o nível máximo de aflatoxina em alimento para animais e de $50 \mathrm{ppb}$ em milho destinado à alimentação animal. Para os países participantes do MERCOSUL (Mercado Comum do Cone Sul) e Estados Unidos, o nível máximo para aflatoxina em alimento é de 20 ppb. Não existe regulamentação quanto ao nível máximo permitido para outras micotoxinas (FONSECA, 2001).

O Programa Nacional para Controle de Micotoxinas foi criado em 1997 e prevê ações para a educação, monitoramento e inspeção de produtos, subprodutos e derivados de origem animal, mas as inspeções e análises do nível das micotoxinas na 
cadeia produtiva não foram iniciadas até o final do ano 2000 (SALAY et al., 2001).

\section{Agradecimentos}

Aparecida Maria de Oliveira (técnica de laboratório) pelo auxílio na realização dos exames toxicológicos.

\section{Conclusão}

Com os resultados obtidos em nosso laboratório nas análises de amostras enviadas durante o período em que ocorreram os fenômenos climáticos el niño e la niña, pode-se projetar a possibilidade de dados semelhantes para as colheitas do ano de 2003, já que estão previstas para este ano de 2002, as ocorrências novamente destes distúrbios metereológicos.

A necessidade do desenvolvimento de novos cultivares mais resistentes aos fungos torna-se mais evidente, tanto para a economia quanto para a saúde pública, pois com as novas pesquisas sendo realizadas nesta área tem-se a cada dia resultados comprometedores da eficiência das micotoxinas em causar patologias graves em plantas e na saúde humana e animal.

As micotoxinas são resistentes aos mais variados processos utilizados para descontaminação, então, como controle, também é necessário o estabelecimento de programas de monitoramento como estratégia para a diminuição do risco de exposição, tanto para humanos quanto para animais.

Uma outra medida a ser adotada, enquanto não for possível produzir um suprimento de alimentos completamente livre de micotoxinas, seria a de promover melhorias no armazenamento e na manipulação de grãos e outros substratos com o intuito de minimizar o crescimento de fungos e conseqüentemente, reduzir o risco de contaminação por estas substâncias tóxicas.

\section{Referências}

BOLSEN, K. K. Vamos falar de silagens: aspectos básicos. Feeding Times, v.4, n.3, p. 22-3,1999.

BRASIL. Ministério da Agricultura. Pecuária e Abastecimento. Portaria n.07, de 9 de novembro de 1988. Baixa os padrões mínimos de matéria-prima destinada à alimentação animal. Disponível em: <http://www.agricultura.gov.br/dfpa/ html/port07.htm>. Acesso em: nov. 2001.

FONSECA, H. Micotoxinas. Disponível em:<http:// www.micotoxinas.com.br>. Acesso em: nov. 2001.

INSTITUTO ADOLFO LUTZ. Métodos para determinação simultânea de aflatoxina $B$ e $G$, ocratoxina $A$, zearalenona e esterigmatocistina. São Paulo, 1996.

LAZZARI, F.A. Fungos. In: Umidade, fungos e micotoxinas na qualidade de sementes, grãos e rações. Curitiba: Paranaset, 1997. cap.2, p.23-38.

INSTITUTO AGRONÔMICO DO PARANÁ. Clima: programa computacional para organização e análise de dados meteorológicos. Londrina, 2001. (Boletim Técnico)

LOPEZ, L. C.; CHRISTENSEN C. M. Effect of misture content and temperature on invasion of stored corn by Aspergillus flavus. Phytopathology, Saint Paul, v.57, p. 588-590, 1967.

NATIONAL TRADE UNION OF ANIMAL FEED INDUSTRIES. NATIONAL ASSOCIATION OF ANIMAL FEED MANUFACTURERS. Perfil da indústria brasileira de alimentação animal. São Paulo, 2000.

MABBETT, T. A ameaça das micotoxinas. Feeding Times, v.4, n.3, p. 4-5,1999.

MOSS, M. O. The environmental factors controlling mycotoxin formation. In: SMITH, J.E.; ANDERSON, R. A. Mycotoxins and animal foods. Boca Raton: CRC Press, 1991. p. 37-56

MULRENNAM, F. Será que o guerreiro será derrotado? Feeding Times, v.4, n.3, p. 4-5,1999.

SABINO, M. Normas e níveis de tolerância de micotoxinas no Brasil, no MERCOSUL e no mundo. In: SIMPÓSIO SOBRE MICOTOXINAS EM GRÃOS, 1999, São Paulo. Anais... São Paulo: Fundação Cargil, 1999. p.183-192.

SALAY, E. et al. Food safety issues in developing nations: a case study of Brazil. In: HOOKER, N.; MURANO, E. A. (Eds). Interdisciplinary food safety research. Boca Raton: CRC Press, 2001. p.87. 
SALAY, E.; MERCADANTE, A. Z. Mycotoxins in Brazilian corn for animal feed: ocurrence and incentives for the private sector to control the level of contamination. Food Control, Surrey, n.13, p.87-92, 2002.

SHANE, S.H. Economic issues associated with aflatoxins. In: EATON, D.L.; GROOPMAN, J. D. (Eds). The toxicology of aflatoxins: human health, veterinary, and agricultural significance. San Diego: Academic Press, 1994. p.513-527.
SOARES, L. M. V.; RODRIGUES-AMAYA, D. B. Survey of aflatoxin, ochratoxin A, zearalenone, and Sterigmatocystin in some Brazilian foods by using multitoxin Thin-Layer Chromatographic Method. Journal of Association of Official Analytical Chemists, Arlington, v.72, n.1, p.22-26, 1989.

VASANTHI, S.; BHAT, R. V. Mycotoxins in foodsoccurrence, health \& economic significance \& food control measures. Indian Journal of Medical Research, Jodhpur, v.108, p.212-224, 1998. 
